\title{
Methicillin-susceptible Staphylococcus aureus from clinical and community sources are genetically diverse.
}

\begin{abstract}
Despite the association of methicillin-susceptible S. aureus (MSSA) with several lifethreatening diseases, relatively little is known about their clinical epidemiology in Malaysia. We characterized MSSA isolates $(n=252)$ obtained from clinical and community (carriage) sources based on spa sequencing and multilocus sequence typing (MLST). The prevalence of several important virulence genes was determined to further define the molecular characteristics of MSSA clones circulating in Malaysia. Among the 142 clinical and 110 community-acquired MSSA isolates, 98 different spa types were identified, corresponding to 8 different spa clonal clusters (spa-CCs). In addition, MLST analysis revealed 22 sequence types (STs) with 5 singletons corresponding to 12 MLST-CCs. Interestingly, spa-CC084/085 (MLST-CC15) ( $\mathrm{p}=0.038)$, spa-non-founder 2 (MLST-ST188) $(\mathrm{p}=0.002)$, and spa-CC127 (MLST-CC1) ( $\mathrm{p}=0.049)$ were identified significantly more often among clinical isolates. spaCC3204 (MLST-CC121) ( $\mathrm{p}=0.02)$ and spa-CC015 (MLST-CC45) ( $\mathrm{p}=0.0002)$ were more common among community isolates. Five dominant MLST-CCs (CC8, CC121, CC1, CC45, and CC5) having clear counterparts among the major MRSA clones were also identified in this study. While the MSSA strains are usually genetically heterogeneous, a relatively high frequency (19/7.5\%) of ST188 (t189) strains was found, with 57.8\% of these strains carrying the Panton-Valentine leukocidin (PVL). Analysis of additional virulence genes showed a frequency of $36.5 \%$ and $36.9 \%$ for seg and sei and $0.8 \%$ and $6.3 \%$ for etb and tst genes, respectively. Arginine catabolic mobile element (ACME) was detected in 4 community isolates only. These represent the first isolates harbouring this gene in an Asian region. In conclusion, MSSA from the Malaysian community and their clinical counterparts are genetically diverse, but certain clones occur more often among clinical isolates than among carriage isolates and vice versa.
\end{abstract}

Keyword: Methicillin-Sensitive Staphylococcus aureus. 